\title{
MasterFinger: Multi-finger Haptic Interface for Collaborative Environments
}

\author{
Mary Monroy, María Oyarzabal, Manuel Ferre, \\ Alexandre Campos, and Jorge Barrio \\ Universidad Politécnica de Madrid, \\ Jóse Gutierrez Abascal 2, 28006 Madrid, Spain \\ \{mmonroy, moyarzabal, mferre, jbarrio, acampos\} @etsii.upm.es \\ http://www.disam.upm.es/grmi/indexen.htm
}

\begin{abstract}
This paper introduces the MasterFinger developemt and application, a multi-finger haptic interface for virtual object manipulation. This haptic device, with a modular interface, is specially designed to perform collaborative tasks. Each module is in charge of managing the haptic interaction with a finger. The mechanical structure of the module is based on a serial-parallel structure linked to the finger thimble by a gimble with its own controller. Cooperative applications based on MasterFinger-2 (MF2) are also described in this study. Results from these applications show that multifinger interface is a significant leap in haptic devices since precise object grasping and collaborative manipulation by using two hands are successfully performed.
\end{abstract}

Keywords: haptic interface, multi-finger interface, collaborative tasks, cooperative tasks.

\section{Introduction}

Haptic interfaces are devices that provide users with force information while they are interacting with virtual objects, allowing them to perform manipulation tasks and cooperate among users. Multi-finger haptic interfaces take advantage of the use of several fingers, thereby processing a large number of degrees of freedom, to improve interaction with virtual environments and increase the sense of immersion.

There are many approaches to haptic interfaces in literature: Phantom [1], Spidar-G [2], Sarcos [3], ViSHard [4], EXOS Force ArmMaster [5], Safire (Sensing and Force Reflecting Exoskeleton) and cyberGlove [6] are some of them. Regarding multifinger haptic devices, there some interesting developments such as Hiro-II [7] and other interfaces that are mostly glove [8] and exoesqueleton structures [9].

This paper introduces a new two-finger haptic interface, called Masterfinger-2, which attempts to improve haptic perception since grasping objects can be easily reproduced by using this device. This interface is based on an open architecture 
that allows controlling each finger independently via Ethernet; therefore, it permits an easy development of co-operative tasks, where users interact directly with their fingers instead of using a tool.

MasterFinger is based on a modular design where each finger has its own mechanical structure and electronic controller. Fingers are inserted into a thimble that has 6 degrees of freedom, therefore any position and orientation can achieved by fingers into the corresponding workspace. Forces are reflected in any direction since there are 3 actuators per finger.

This paper is organized as follows; next section describes the mechanical design of the haptic interface. Section three is focused on the development of haptic applications by using the MasterFinger. Section four discusses the main conclusions of this work.

\section{MasterFinger Design}

MasterFinger is a modular haptic interface where each finger is independently managed. All modules have the same mechanical structure and controller. Therefore, it is easy to scale the system from one to three fingers. Next section shows the mechanical design of MasterFinger modules and describes versions for two and three fingers.

\subsection{Design of MasterFinger Modules}

Each finger is treated as an independent module with its own mechanical structure, controller and communications. Mechanical module design is based on a serial-parallel structure that confers it a wide workspace with very low inertia.

This configuration allows a comfortable manipulation of the haptic interface since actuator inertia is mainly supported by the base.

A module consists of a 6-degree of freedom mechanism and 3 actuators as it is shown in Fig. 1. The second and third actuators are linked to a 5-barstructure that provides a wide workspace area. This structure is linked to a

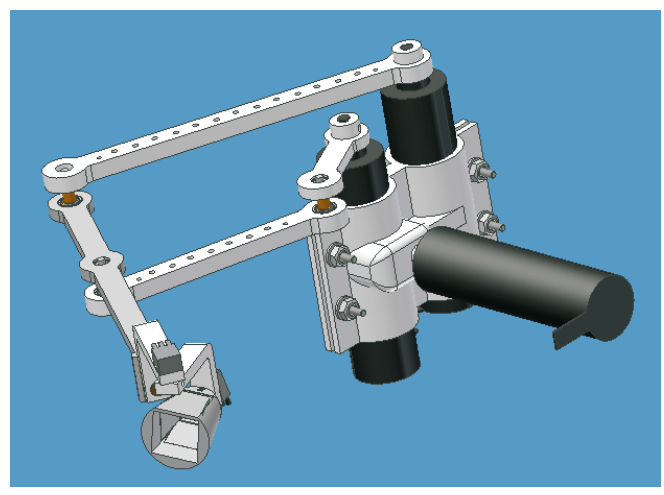

Fig. 1. Master Finger Basic Module 


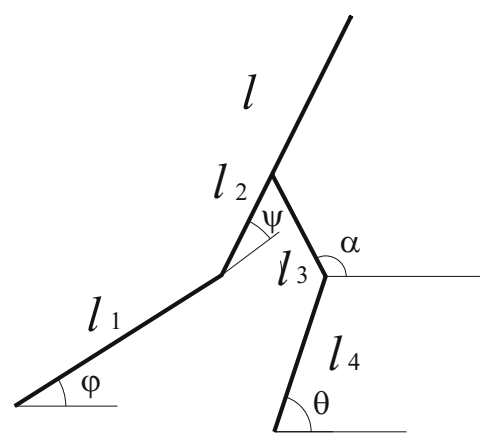

Fig. 2. Schematic view of the two first degrees of freedom

thimble by a gimble with 3-rotational degrees of freedom. The first degree of freedom allows the vertical hand movement (approximately corresponding to the deviation movement ulna-radius in the wrist) while the second and third degrees of freedom are mainly associated to the movement of the finger. Figure 2 shows the five-bar-mechanism which is formed by the parallel structure (second and third degrees of freedom).

Equations describing the 5 bar-mechanism are:

$$
\begin{aligned}
& x_{p}=l_{1} \cos (\varphi)+l \cos (\varphi+\psi) \\
& y_{p}=l_{1} \sin (\varphi)+l \sin (\varphi+\psi)
\end{aligned}
$$

$\varphi$ and $\theta$ angles are provided by the encoders, and $\alpha$ and $\psi$ can be calculated according to equations in Annex I.

Thimble orientation is measured by 3 encoders which are placed in the corresponding gimble joints. Figure 3 shows details of thimble and gimble. The thimble can be orientated in any direction in order to guarantee free movements of the finger. The three rotational axis of gimble intersect in the user's finger

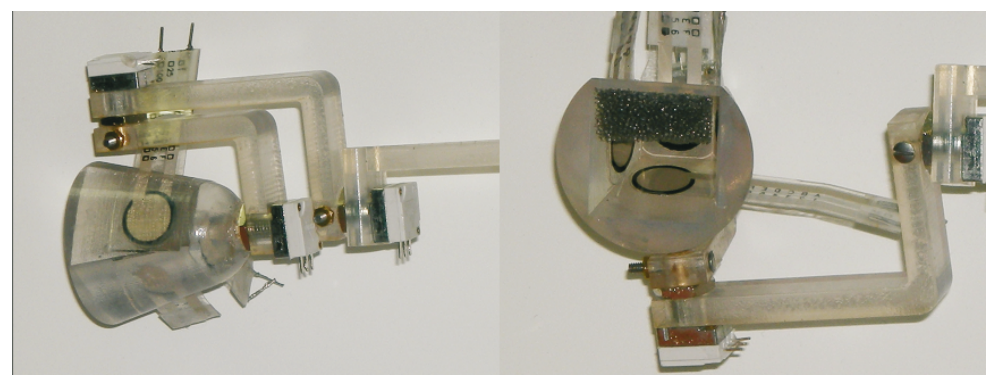

Fig. 3. Lateral and frontal view of the thimble and the sensors 
tip. This geometrical configuration avoids torque reflection, that is, only forces are reflected to the user finger. Thimble has been developed to enclose completely the operator's finger. Thimble includes 4 Flexiforce sensors by Tekscan Inc. These sensors are used to estimate normal and tangential forces exerted by the user. Normal forces are obtained from the sensor placed at the bottom of the thimble in contact with the finger tip. Tangential forces are estimated from sensors placed on the lateral faces of the thimble. Figure 3 shows two views of the thimble with these sensors.

Regarding interface components, all parts of the MasterFinger v1.0 are built using stereo-lithography, a technique of rapid prototyping, and the material used is epoxy resin. It has the advantage of a low weight that allows an easy manipulation of all the elements making up the interface. Reflected forces are generated by Maxon DC motors (RE 25, 10W) with a planetary gear unit GP26 with a $225 / 16$ reduction. These motors also include a 1000-pulse-per-revolution encoder providing motor orientation.

\subsection{MasterFinger-2 Haptic Interface}

MasterFinger-2 is made up of two modules, placed in such a way that the index and thumb fingers can handle it. It allows the user to interact with virtual environments in an easy and comfortable manner for grasping tasks. Both modules are connected to the interface base with an additional joint to increase the workspace of this haptic interface. The first motor of both modules is in a horizontal plane; therefore, device inertia is significantly reduced. Figure 4 shows a general view of MF-2.

Figure 5 shows the workspace of MasterFinger-2, it is close to a sphere with a radius of $15 \mathrm{~cm}$. This space represents the area where index and thumb finger tips can be located, therefore hand movements correspond to a higher space.

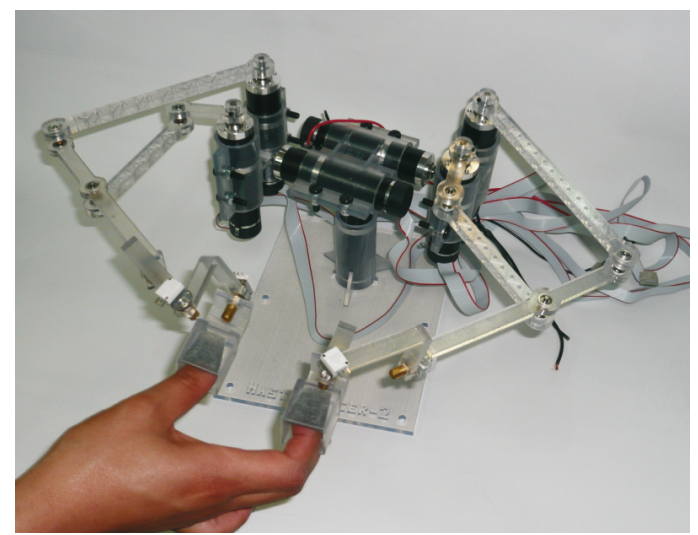

Fig. 4. MasterFinger-2: six degrees of freedom for each fingers 